P4-022 APATHY NEUROCIRCUITRY: BRAIN IMAGING FINDINGS AND IMPLICATIONS

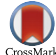

David L. Sultzer, David Geffen, School of Medicine at UCLA, Los Angeles, CA, USA. Contacte-mail: dsultzer@ucla.edu

Background: Apathy is the most common neuropsychiatric symptom in Alzheimer's disease (AD), occurs early in the disease process, and is associated with poor cognitive and functional outcomes. The neurobiological underpinnings for the expression of apathy in $\mathrm{AD}$ are not clearly understood and can be assessed via neuroimaging techniques to reveal key mechanisms and treatment targets. Methods: 1. Apathy was assessed in 87 individuals with mild to moderate $\mathrm{AD}$, using the SANS-AD to measure each of the specific cognitive, functional, and emotional domains of apathy. FDG-PET neuroimaging was used to assess the pattern of hypometabolism associated with each apathy domain. 2. Global and individual domains of apathy were measured in 29 individuals with AD. 2FA neuroimaging was used to measure regional $\alpha 4 \beta 2$ nicotinic cholinergic receptor binding and to assess relationships between cholinergic receptor binding and the extent of global and domain-specific apathy. 3. A literature review of neuroimaging studies of apathy in AD was performed. Results: Structural and functional imaging studies using MRI, FDG-PET, or perfusion SPECT typically revealed alterations in anterior cingulate, orbitofrontal cortex, and insula associated with apathy in AD. Other frontal regions, temporal cortex, or subcortical nuclei were implicated in some studies, and there was overlap with regions linked to poor insight. Frontal white matter lesions and loss of integrity of white matter tracts on DTI images were also relevant. Associations in MCI were less clear. Apathy was associated with increased global or regional amyloid ligand binding. Each of the three apathy domains was associated with hypometabolism in a unique set of brain regions in $\mathrm{AD}$. Greater apathy was correlated with lower cholinergic binding in middle cingulate and lateral orbitofrontal cortex. Across the neuroimaging studies, relationships were not attributable to depression. Conclusions: Neuroimaging studies indicate that altered structure, function, or neuroreceptor binding in a frontal-limbic-subcortical neural system are associated with apathy in AD. Specific regional alterations drive distinct aspects of apathy and neurotransmitter levels may influence overall tone. Disease-related alterations across this neural system likely promote dysfunction in directed attention, stimulus salience assessment, emotional processing, affective drive, and reward-related decision-making that emerge as clinical apathy in AD.

\section{P4-023 HIGH-DEFINITION TRANSCRANIAL DIRECT CURRENT STIMULATION MODULATES BOLD SIGNAL DURING SPATIAL NAVIGATION IN OLDER CONTROLS AND PATIENTS WITH MCI}

Benjamin M. Hampstead ${ }^{1,2}$, Alina Lesnovskaya ${ }^{2}$, Sarah Garcia $^{2}$, Sean $\mathrm{Ma}^{2}$, Arijit K. Bhaumik ${ }^{2}$, Bruno Giordani ${ }^{2}$, Scott Peltier ${ }^{2},{ }^{1}$ VA Ann Arbor Healthcare System, Ann Arbor, MI, USA; ${ }^{2}$ University of Michigan, Ann Arbor, MI, USA. Contact e-mail: bhampste@med.umich.edu

Background: Navigation is critical in daily life and can be accomplished using allocentric and/or egocentric systems. Allocentric navigation utilizes an external reference system and cognitive maps that are preferentially mediated by spatial processing (e.g., parietal cortex) and episodic memory (e.g., hippocampus) brain areas. Egocentric navigation uses an internal reference system and stimulus-response relationships that are preferentially mediated by the striatum (e.g., caudate nucleus). "Normal" age-related decline is evident in the allocentric, but not egocentric, processing and is exacerbated in those with mild cognitive impairment (MCI). Transcranial direct current stimulation (tDCS) may hold promise for enhancing functioning due to its neuromodulatory effects. The current study evaluated the behavioral and neurophysiological effects of high-definition tDCS in cognitively intact older adults (HOA) and patients with MCI. Methods: Using a double-blind cross-over design, 36 participants (18 MCI) each completed two sessions wherein they were randomized to active or sham HDtDCS (2mA for 20 minutes) that targeted the right superior parietal lobule (center anode over site P2 in 10-10 system). Participants underwent functional magnetic resonance imaging (fMRI) immediately after stimulation, during which they encoded a series of allocentric and egocentric environments. Memory was evaluated after the scan session. Results: While there were no effects of HD-tDCS on memory accuracy (likely related to task difficulty), there was a group by session interaction on reaction time where HOA were slower but MCI were faster to respond following stimulation. Preliminary fMRI analyses revealed main effects of group (greater caudate activation in HOA) and session (increased activation in the left superior parietal lobule but decreased activation in the anterior cingulate following HD-tDCS). Group by session effects were evident in a number of left hemisphere regions associated with successful encoding (e.g., hippocampus, insula, lateral prefrontal cortex) and bilateral rostral prefrontal cortices that may reflect increased top-down control. Conclusions: This double blind RCT revealed offline behavioral and neurophysiologic effects of HD-tDCS that differ as a function of cognitive phenotype (HOA vs. MCI). Findings add to knowledge of individual differences in tDCS response, support age- and disease-related compensatory patterns of activation, and hold promise for more extensive neurorehabilitative approaches that target this vital cognitive ability.

\section{P4-024 DESIGN AND EXECUTION OF THE NILVAD STUDY}

Brian Lawlor, Medical Gerontology, School of Medicine, Trinity College, Dublin, Ireland. Contact e-mail: blawlor@stjames.ie

Background: NILVAD is an investigator driven phase III clinical trial of nilvadipine in mild to moderate Alzheimer's disease. Nilvadipine, a dihyropyridine calcium channel blocker, is a licensed antihypertensive and is being repurposed for AD based on clinical and basic science evidence for its potential as a disease-modifying agent in AD. Methods: 511 subjects with mild to moderate AD were recruited across 23 sites in 9 different European countries. Subjects received $8 \mathrm{mg}$ over encapsulated nilvadipine or matching placebo over a period of 18 months. The sponsor of the trial was St. James's Hospital, Dublin. Academic trial units were used to monitor the trial in each country. The primary outcome measures were Alzheimer's disease Assessment Scale Cognitive (ADAS-Cog) and Clinical Dementia Rating Scale sum of boxes (CDR-sb). Results: There were a number of challenges to overcome in successfully completing this trial including: recruitment and exclusion of patients on multiple antihypertensive agents, in particular other calcium 